\title{
Facilitators and barriers to the privacy of Iranian hospitalized patients in
}

\section{government hospitals: A qualitative content analysis}

Tayebeh Hasan Tehrani ${ }^{1}$, Sadat Seyed Bagher Maddah², Masoud Fallahi-Khoshknab², Farhnaz Mohammadi Shahbooulaghi ${ }^{1}$, Abbas Ebadi $^{3}$, and Mark Gillespie ${ }^{4}$

1. Department of Nursing, Iranian Research Center on Aging, University of Social Welfare and Rehabilitation Sciences, Tehran, Iran

2. Department of Nursing, University of Social Welfare and Rehabilitation Sciences, Tehran, Iran

3. Behavioral Sciences Research Center and Nursing Faculty, Baqiyatallah University of Medical Sciences, Tehrani, Iran

4. Department Mental Health Practice and Psychosocial Interventions, University of the West of Scotland, Scotland

\section{RESEARCH}

Please cite this paper as: Hasan Tehrani T, Seyed Bagher Maddah S, Fallahi-Khoshknab M, Mohammadi Shahboulaghi F, Ebadi A, Gillespie M. Facilitators and barriers to the privacy of Iranian hospitalized patients in government hospitals: A qualitative content analysis. AMJ 2018;11(2):74-77. https://doi.org/10.21767/AMJ.2017.3248

\section{Corresponding Author:}

Masoud Fallahi-Khoshknab

Department of Nursing, University of Social Welfare and Rehabilitation Sciences, Koodakyar St, Daneshjoo Ave, Evin, Tehran, Iran

Email: fallahi@uswr.ac.ir

\section{ABSTRACT}

\section{Background}

Privacy is one of the essential needs of humans, and is therefore crucial in effective healthcare systems. There is limited research clearly addressing the facilitators and barriers underpinning privacy for hospitalized patients.

\section{Aims}

This study aimed to explore Iranian patients' perceptions and experiences with the facilitators and barriers of privacy in government hospital in-patients.

\section{Methods}

This qualitative study was conducted on 22 patients admitted to the in-patient departments of government hospitals in Tehran, Iran, in 2016. The study population was selected through purposive sampling technique. The data were collected using individualized semi- structured interviews, which were recorded and transcribed. Data analysis was performed by means of an inductive content analysis approach.

Results

The analysis of the experiences of the hospitalized patient's extraction of six themes. These themes included the ethical and legal backgrounds of privacy, multi-dimensional design of privacy, perceived vulnerability, patriarchal care, conflict between privacy and care, individual backgrounds, nature of disease, and informed consent.

\section{Conclusion}

The privacy of the hospitalized patient is affected by complicated factors. These factors are facilitators and barriers and should be considered in the design of care.

\section{Key Words}

Privacy, patients, hospitalization, facilitators, barriers

\section{What this study adds:}

\section{What is known about this subject?}

The privacy of the hospitalized patient is affected by complicated factors, which ultimately affects the satisfaction and quality of care.

\section{What new information is offered in this study?}

In this study, the factors affecting the privacy of patients from their own point of view were identified. 
3. What are the implications for research, policy, or practice?

The evaluation of patient privacy in healthcare centres can facilitate the establishment of an operational model of patient privacy and development of the native tools.

\section{Background}

Respect for privacy is one of the most basic rights of human beings. Privacy is an important concept in the areas of morality, care, and nursing. ${ }^{1}$ Moreover, this concept has been emphasized by a multitude of international organizations and associations as core within their codes of conduct and essential standards of care. ${ }^{2-4}$

Accordingly, In Iran, privacy is considered as one of the most important terms in the patients' rights charter. ${ }^{5}$ The majority of studies that have been performed around the experience of privacy for patients have reported weak to medium patient satisfaction with the respect for their privacy shown. ${ }^{6,7}$

The results of quantitative studies showed that patients have experienced many problems with privacy in the hospital, despite the high emphasis on patient privacy. There is still little interest in the privacy of patients. ${ }^{8-10}$ Some of these behaviors have become so commonplace for the healthcare provider that the treatment team does not even think their behavior is a violation to the patient's privacy. ${ }^{11}$

To the extent of the researchers' knowledge, there are no studies, comprehensively evaluating the facilitators and barriers to respecting the privacy of hospital in-patients. This issue has been only marginally indicated in the studies completed, leading to some recommendations being made for the improvement of patient privacy and respect.

The importance of the topic and the relative lack of research focus it has been afforded is concerning, and emphasizes a need to explore patient privacy further. It seems that the most reliable method to assess privacy in care is to directly evaluate this from the patients' perspective. ${ }^{12}$ Iven the fact that the meaning of privacy depends on the culture, religion, and individual priorities of the patients involved. ${ }^{13}$ This study aimed to determine the effective facilitators and barriers to respecting the privacy of hospitalized patients in Iran.

\section{Method}

This qualitative study was conducted with Twenty-two patients 20-65 years were interviewed that admitted to the in-patient departments of government hospitals in Tehran, Iran, in 2016, that was selected through purposive sampling technique and the sampling continued until data saturation.

The data collection was performed using semi-structural interviews. Some of the questions asked in this stage were as follows: "what experiences did you have during the care process?", "please, explain about your experiences of carer visits?" Data analysis was carried out using MAXQDA software after typing the interview.

Naming of the codes was performed with the continuation of this process. Subsequently, based on the similarities and differences between the codes, and subcategories, they were combined to create a main category. In addition, Lincoln and Guba's four evaluative criteria were used to determine the validity and reliability of the qualitative study.

\section{Results}

The analysis of the data, were categorized under six themes (Table 1). In the majority of the cases, the patients prioritized the ethical behaviors of the healthcare team over their skills and care. In this regard, one of the participants pointed out, "When the nurses are going to be recruited, before their nursing skills, their ability to deal with patients should be taken into account." Participant 2.

According to the participants, many factors were involved in receiving respect for privacy, some of which were assigned into a theme termed multi-dimensional design of privacy. Environmental cleanness in the hospital departments was one of the topics noted by 100 per cent of the patients. In this regard, a subject stated, "Personally, I think cleanness is really important. Something that bothered me most was the poor cleaning of the ward and my room, which is the worst factor since it causes infection" Participant 5. The design and architecture of the hospital wards were also other points indicated by the patients; "According to the experiences of the patients, the organizational structure of the hospitals played a significant role in the preservation of their privacy. In terms of the collaboration between the physicians and healthcare staff, one of the Most of the patients pointed out that the medical team did not provide them with adequate responses to questions around various aspects of treatment or care. In this regard, one of the participants specified, "Here, when you ask a question to a healthcare provider, they say that they have no information in this regard and that I have to ask my physician. I would like to know about my treatment process, and I know that if 
they clearly explain the procedures to me, I will tolerate my pain Better" Participant 16.

The participants had a sense of vulnerability in the hospital due to the hospital hierarchy and reduction of their sense of control over their lives. In this respect, one of the subjects suggested, "Currently, when a patient is insulted, they advocate for their colleague. They think that if they warn their colleague in front of the patient, the patient would become impudent, and his/her expectations would increase. Regulations have been replaced with partisanship, and no one works within the specified framework" Participant 21.

The experiences of the participants were indicative of a conflict between care and privacy. One of the subjects argued, "It is like we are kept in a barracks. There is a nurse who slams the door and turns on all of the lights."

Obtaining informed consent is closely associated with respecting the privacy of hospitalized patients. In the present study, the majority of the participants expected to obtain information about various therapeutic interventions, allowing them to be able to make informed decisions and have control over various aspects of their privacy.

\section{Discussion}

The majority of the patients believed that the ethical values of the treatment team were prioritized over care quality. The majority of experts believe that the concepts of professional ethics and patient's rights must be included in the educational contents of healthcare professions. ${ }^{14,15}$

The multi-dimensional design of privacy was another important factor for respecting the privacy of the hospitalized patients. Mozafari et al, evaluated the necessities of establishing a patients' rights, the main areas emphasized by the experts included physical space, gender segregation, the diagnosis and treatment environment, and provision of proper restrooms for patients. ${ }^{14}$ The subjects identified that privacy is occasionally sacrificed for the sake of care. The healthcare team sometimes sacrifices the privacy of the patient in order to avoid environmental dangers and save their lives. ${ }^{16}$ It is essential to prepare and inform patients adequately, to decrease their dissatisfaction with any necessary violation of their privacy. ${ }^{17}$

The final component influencing the respect for the privacy of patients was informed consent. The subjects believed that the health providers must obtain patients' permissions for using their personal information if they want to respect patient privacy. ${ }^{5}$
The limitation of this study was the participants' potential fear of expressing their real experience. The findings of this study can help healthcare managers better detect the available opportunities and establish the proper foundation for respecting the privacy of in-patients and facilitate the establishment of an operational model of patient privacy policy and development of the native tools evaluating this concept within healthcare services.

\section{Conclusion}

In order to respect the privacy of the hospitalized patients, it is essential to implement some changes in the structure and performance of healthcare centers. According to the results of the current study, improved respect for hospitalized patient privacy could be accomplished through the modification of management and processes as well as empowerment of the human resources available. Given that the present study was conducted in one specific area, incorporating only the experiences of participants within this location, it is recommended that similar studies be conducted in other environments to increase the generalizability and application of the results.

\section{References}

1. Leino-Kilpi $H$, Välimäki $M$, Dassen $T$, et al. Privacy: a review of the literature. Int J Nurs Stud. 2001;38(6):66371.

2. Association. AM. Code of medical ethics of the American Medical Association [Adobe Digital Editions version] www.ama-assn.org/ama/pub/physicianresources/medical-ethics/codemedical- ethics/codemedical-ethics.2015.

3. Association AN. Code of ethics for nurses with interpretive statements: Retrieved from www.Nursebooks.org, ePDF: 978-1-55810-600-0 2015.

4. Nurse Ic. Code of Ethics for Nurses ICN 2012 [cited 17 may 2017]. Available from: http://www.icn.ch/who-weare/code-of-ethics-for-nurses/.

5. Parsapoor A, Bagheri A, Larijani B. Patient rights in Iran. Med Ethics` History. 2009;27(special issue):39-47.

6. Hajbaghery MA, Chi SZ. Evaluation of elderly patients' privacy and their satisfaction level of privacy in selected hospitals in Esfahan. J Med Ethics. 2015;8(29):97-120.

7. Moore M, Chaudhary R. Patients' attitudes towards privacy in a Nepalese public hospital: a cross-sectional survey. BMC Res Notes. 2013;6:31.

8. Yazdanparast E, Davoudi M, Ghorbani SH, et al. The observance of different aspects of patient privacy: Analysis of elderly views. Med Ethics J. 2016;10(36):7380. 
9. Rasti R, Jahanpour F, Ravanipour M, et al. Evaluation of patients' views about personal privacy during nursing care. 2014;54-63.

10. Nayeri ND, Aghajani M. Patients' privacy and satisfaction in the emergency department: a descriptive analytical study. Nurs Ethics. 2010;17(2):167-77.

11. Baillie L. Mixed-sex wards and patient dignity: nurses' and patients' perspectives. Br J Nurs. 2008;17(19):12205.

12. Swan M. Emerging patient-driven health care models: an examination of health social networks, consumer personalized medicine and quantified self-tracking. Int J Environ Res Public Health. 2009;6(2):492-525.

13. Serenko N, Fan L. Patients' perceptions of privacy and their outcomes in healthcare. Int J Behav Healthc Res. 2013;4(2):101-22.

14. Mozafari M, MOUSAVI MS, Jafari A. Surveying the prerequisites for establishing patient's rights charter in health centers of iran-a Delphi study. J of Clinil Nurs and Midwif. 2013;2(3):74-85.

15. Johnson A, Shmatikov V. Privacy-Preserving Data Exploration in Genome-Wide Association Studies. KDD: proceedings/ International Conference on Knowledge Discovery \& Data Mining International Conference on Knowledge Discovery \& Data Mining. 2013;2013:107987.

16. Leigh $\mathrm{H}$. The patient: Biological, psychological, and social dimensions of medical practice: Springer Science \& Business Media; 2013.

17. Greene J. Behavioral health data in the electronic health record: privacy concerns slow sharing. Ann Emerg Med. 2013;62(4):19A-21A.

\section{ACKNOWLEDGEMENTS}

This article was a part of a doctoral dissertation submitted by first author. Hereby, we extend our gratitude to all participants and the Research Deputy of the University of Social Welfare and Rehabilitation Sciences for their cooperation with the study.

\section{PEER REVIEW}

Not commissioned. Externally peer reviewed.

\section{CONFLICTS OF INTEREST}

The authors declare that they have no competing interests.

\section{FUNDING}

The University of Social Welfare and Rehabilitation Sciences.

\section{ETHICS COMMITTEE APPROVAL}

The Research Council of the University of Social Welfare and Rehabilitation Sciences with the ethical confirmation code of IR.uswr.rec1394.382.

Table 1: Main themes and subthemes of effective facilitators and barriers to the privacy of Iranian hospitalized patients

\begin{tabular}{|l|l|}
\hline Main themes & Subthemes \\
\hline $\begin{array}{l}\text { Ethical and legal } \\
\text { backgrounds of privacy }\end{array}$ & $\begin{array}{l}\text { Ethical rights of privacy/ Patient's } \\
\text { familiarity with their rights }\end{array}$ \\
\hline $\begin{array}{l}\text { Multi-dimensional } \\
\text { design of privacy } \\
\text { Equipment and facilities of the } \\
\text { ward/ Organizational structure/ } \\
\text { pragmatic and scientific } \\
\text { qualification of personnel/ } \\
\text { Accountability of healthcare } \\
\text { team/ Preservation of privacy in } \\
\text { clinical education/ Privacy- } \\
\text { oriented design }\end{array}$ \\
\hline $\begin{array}{l}\text { Perceived vulnerability } \\
\text { and patriarchal care }\end{array}$ & $\begin{array}{l}\text { Hospitals hierarchy/ Decreased } \\
\text { patient control }\end{array}$ \\
\hline $\begin{array}{l}\text { Conflict between } \\
\text { privacy and care }\end{array}$ & $\begin{array}{l}\text { Incomplete routines of privacy/ } \\
\text { Sacrifice privacy to care/ Privacy } \\
\text { sensitivity in specific groups } \\
\text { sensitivity Privacy in private care }\end{array}$ \\
\hline $\begin{array}{l}\text { Individual backgrounds } \\
\text { and nature of the } \\
\text { disease }\end{array}$ & $\begin{array}{l}\text { Individual factors affecting } \\
\text { privacy/ Condition of patients } \\
\text { and diseases }\end{array}$ \\
\hline Informed consent & $\begin{array}{l}\text { The right to obtain information/ } \\
\text { Informed decisions }\end{array}$ \\
\hline
\end{tabular}

